\title{
Research Paper: Impact of SHOFER Computer Game on Visual-Motor Coordination in Children With Hearing Impairments
}

\author{
Zahra Norouzi Tabrizi Nejad' ${ }^{1}$ (D), Rita Rezaee ${ }^{2 *}$ (D), Seyed Alireza Derakhshanrad ${ }^{3}$ (D), Ali Mohammad Hadianfard ${ }^{4}$ (D) \\ 1. Department of Health Information Technology, School of Management and Medical Information Sciences, Shiraz University of Medical Sciences, \\ Shiraz, Iran. \\ 2. Health Human Resources Research Center, Shiraz University of Medical Sciences, Shiraz, Iran. \\ 3. Department of Occupational Therapy, School of Rehabilitation Sciences, Shiraz University of Medical Sciences, Shiraz, Iran. \\ 4. Department of Health Information Technology, School of Allied Medical Sciences, Ahvaz Jundishapur University of Medical Sciences, Ahvaz, Iran.
}

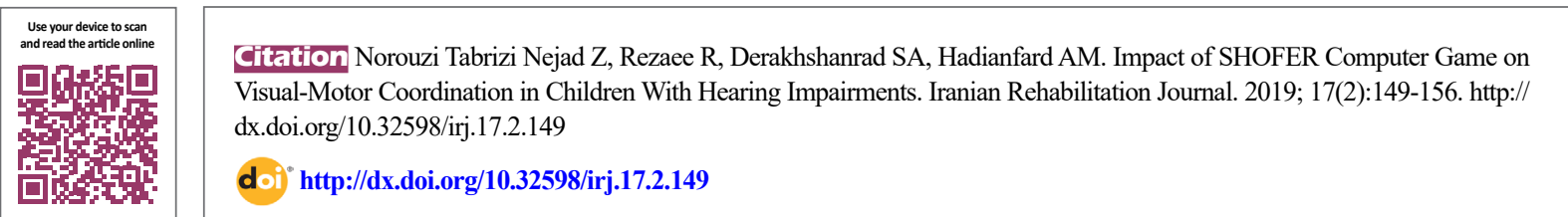

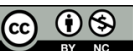

Article info:

Received: 23 Sep 2018

Accepted: 15 Jan 2019

Available Online: 01 June 2019

\section{Keywords:}

Attention, Visual motor coordination, Video games

\section{ABSTRACT}

Objectives: The current study aimed at investigating the impact of SHOFER computer game on visual-motor coordination in children with hearing impairments.

Methods: The current pre-test, post-test experimental study was conducted in Ahvaz, Iran in 2018. Sixteen preschool children with hearing impairment were randomly assigned into two groups of experimental $(n=8)$ and control $(n=8)$. The experimental group subjects played the SHOFER computer game (driving/racing genre) two 45-minute sessions per week for a five consecutive weeks, but the control group did not receive any intervention. Before and after the intervention, visual perception and attention, as visual-motor coordination components, were measured by the advanced Frostig test of visual perception and continuous performance test in both groups. Using SPSS version 21, the performance of the two groups was compared by running the Mann-Whitney and Wilcoxon tests.

Results: The analysis of findings with the effect size indicator showed that the computer game increased attention and spatial perception in the experimental group. However, no significant difference was found between the groups, except in the sub-test of figure-ground perception.

Discussion: The present study showed that commercial computer games can be used to improve visual-motor coordination of children with hearing impairments.

* Corresponding Author: 


\section{Highlights}

- SHOFER computer game might be useful in improving visual-motor coordination of children with hearing impairments.

- The impact of SHOFER game on visual-motor coordination could be due to the game characteristics including speed, variety of objects to follow, and the emphasis on environmental processing.

\section{Plain Language Summary}

Recently, there came a wave of interest in using computer/video games as a valuable, ecologically valid, and cost-effective treatment tool known appealing to children. It is reported that the incorporation of these games in rehabilitation can offer children meaningful experiences in such a way as to bring them visual-motor-related benefits. Since hearing impairment is associated with motor incoordination, it was hypothesized in the present study that using a computer game would provide children with hearing impairment the opportunity to improve their visual-motor coordination. As a result, in the current study, the authors applied a computer game called SHOFER with driving/racing genre to examine its effectiveness on visual-motor coordination of a sample of children with hearing impairments.

\section{Introduction}

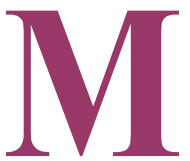

ore than $5 \%$ of the world's population, or about 466 million people, have some kinds of hearing impairments, of those 34 million are children [1]. Children with hearing problems are at risk of motor problems [2]. Motor performance deficits in such children can lead to motor incoordination, especially with regard to performing complex motor tasks [3]. Visual-motor incoordination is such a motor problem in preschool and primary school children with hearing impairments that can lead to detrimental effect on learning sign language, which is the communication language of the deaf [4-6].

Vision is used to guide body movements. Accordingly, visual-motor coordination refers to the ability to control hand movements by visual guide [7]. The prerequisites for this ability are components including visual perception and visual attention. This ability plays an important role in the precise and accurate performance of the individual's daily activities such as closing the buttons $[8,9]$.

In recent years, there is an increasing interest in using computer or video games for the healthcare promotion purposes. Therapeutic results vary depending on the type of games used and characteristics of the intervention groups. Computer games such as computer shooting and driving/ racing games require attention and rapidity in control and reaction to the game. Those games are reported to have significant impacts on visual-motor skills [10]. The driving game also is effective in improving sustained attention, consciousness, and working memory skills through the need for quick response and vehicle control in the middle of a road $[11,12]$. Other advantages include the ability to solve problems and the development of social and spatial capabilities [13-15]. Studies conducted to evaluate the effects of such games on various aspects of health suggest using them as an exciting therapeutic activity that utilizes motivational capabilities [16].

Although extensive research are performed to show the feasibility of computer games as an independent therapeutic tool or as an indirect treatment by making therapeutic goals more attractive [16], very few studies on children with hearing impairment showed the effectiveness of such games. Therefore, the current study aimed at evaluating the implication of a computer game to improve visual-motor coordination in children with hearing impairment. The computer game was used in the current study design since it is believed that children with hearing impairments are visually-dependent, so it might be possible that computer games, with so many visual-motor-related features, are appealing to those children; thus, can improve some aspects of their visual-motor coordination [17]. More specifically, the study examined the effectiveness of SHOFER computer game with driving/ racing genre on visual-motor coordination of a sample of children with hearing impairments.

\section{Methods}

\section{Study setting and participants}

The current pre-test, post-test experimental study was conducted to investigate the impact of a computer game 
on visual-motor coordination. Convenience sampling was performed among the students of a special school serving students with hearing impairments. There were 17 students in total, but one of them was not included due to the lack of participation in pretest. Sixteen subjects were divided into two experimental and control groups ( $\mathrm{n}=8$ in each group). Inclusion criteria were as follows: 1. Being in preschool student; 2 . Not having the experience of playing the SHOFER prior to the study; 3 . Having severe or deep hearing impairments.

\section{Intervention (SHOFER computer game)}

SHOFER is a racing and driving game, compatible with Iranian culture due to the Iranian environment and cars used in this game. SHOFER is designed by the Iranian company, Zhouri Maang and has a release confirmation from Steam Company [18]. This game is appropriate for three-year-old children and above according to the Entertainment Software Rating Association (ESRA) that evaluates games in terms of violence, fear, and other harmful contents [19]. SHOFER can be played individually and has different degrees of difficulty (from easy to hard level).

The player can enter into more difficult stages after gaining success at primary stages. One of the features of SHOFER is the continuous competition with other cars, a process in which the player never gets too far behind or far ahead of other cars, in order to keep the competition exciting. Also, after winning the races, new cars are awarded to the player, which can be considered effective in the game's attractiveness. The player must control the car among other vehicles, to win the competition [18]. See Figure 1 for a screenshot of this game.

In a review study of articles employed serious games in rehabilitation of children with sensory-motor impairments, the usual duration of interventions was four weeks (ranged three to twelve weeks) with the average

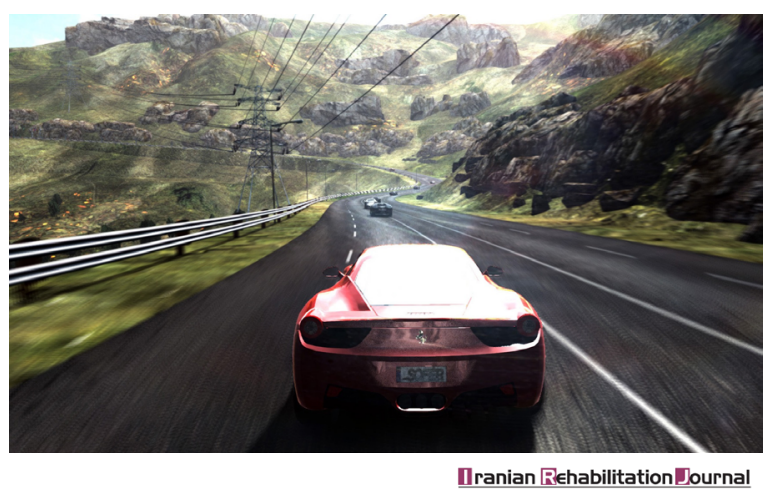

Figure 1. Screenshot of SHOFER computer game frequency of three sessions per week (ranged one to five sessions per week), with an average time of 60 minutes per session (ranged 15 to 90 minutes) [15]. Therefore, according to the school's conditions and the children's educational period, the experimental group played the computer game for five weeks/two 45-minute sessions per week, two-by-two on two separate laptops. During the intervention, the researcher directly monitored each player's playtime. Missing sessions were compensated as soon as possible. The control group did not receive any intervention and only were used for comparison.

\section{Research instruments}

In order to assess visual-motor abilities, two common tests including the Frostig test and Continuous Performance Test (CPT) were used. The Frostig developmental test of visual perception is a paper-based test that includes five subtests for measuring the eye-motor coordination, and perception of Figure-ground, form constancy, position in space, and spatial relationships [20]. The test-retest reliability for the mean total score and subtests ranged 0.69 to 0.98 . The split-half method for the mean total score and subtests showed the coefficients between 0.78 and 0.89 [21].

CPT is a computer-based test. In this test, visual stimuli are presented for a short time on a computer screen, and the subject must respond to the target stimulus by pressing one of the keyboard keys. There are 150 Persian characters or figures as stimuli in the test, of which 30 stimuli are considered as the target stimulus. CPT is used to measure attention and impulsive behaviors. The variables obtained by the employment of this test were the number of correct answers, the number of non-response to the target stimulus, and the number of responses to the non-target stimulus. Test-retest reliability was 0.93 and its validity was confirmed via the criterion validity by comparing the normal and attention-deficit/hyperactivity disorder groups [22].

\section{Procedure}

In the current study, the participants were randomly assigned to two groups using the permuted-block randomization with four individuals in each block; the control $(n=8)$ and intervention $(n=8)$ groups. First, the score of visual perception in both groups was measured using the Frostig developmental test of visual perception by an occupational therapist, and under the researcher's supervision in each group. Then, the score of sustained attention was measured for each subject individually using a computerized continuous performance test by the researcher. 


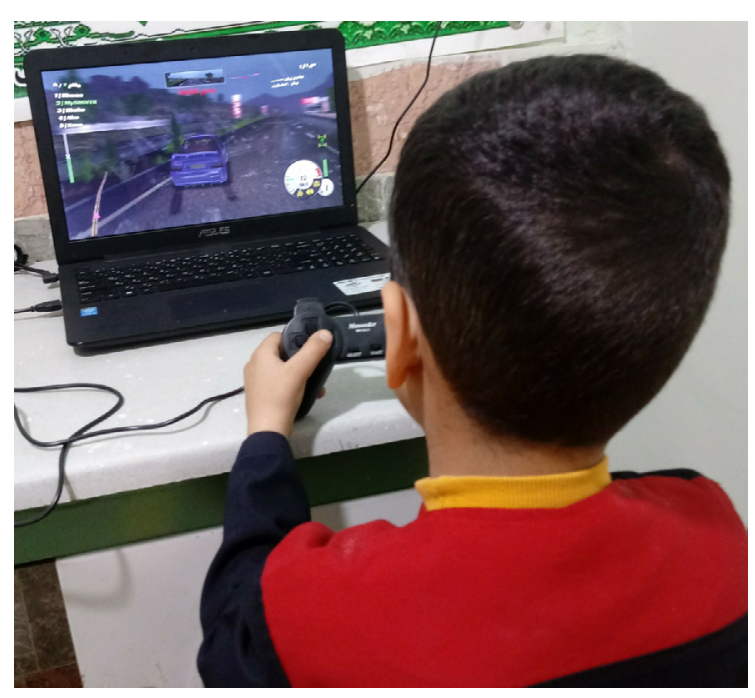

Iranian Rehabilitation Journal

Figure 2. Playing SHOFER computer game

At different times, the researcher focused on learning the sign language and how to communicate with children having hearing impairments by attending school classes. The game model and how to use the joystick were taught individually to each member of the experimental group for 15 to 30 minutes, according to the learning ability of each child.

In order to prevent children from communicating and affecting each other, the game was conducted in a separate room with the presence of the researcher. To ensure that the control group did not play the game, the game was only installed on the researcher's laptops. At first, an easy level was offered to the children. Children could attempt as long as they passed the easier steps of the game (Figure 2). Depending on the subjects' ability and the amount of success they achieved during the 10 sessions, the game level was enhanced from easy to hard. Children's feedback on the offered game was recorded by the researcher.

At the end of the $10^{\text {th }}$ session, the completion of the intervention, visual perception and the sustained attention of both experimental and control groups were evaluated using the Frostig developmental test of visual perception and CPT, and the pretest-posttest results were compared for each subject. The overall results of both groups were compared to examine the effect of the intervention.

\section{Data analysis}

The Mann-Whitney nonparametric tests were used for intergroup comparisons, and the Wilcoxon for the intragroup comparisons. Due to the small sample size, Cohen's effect size index (Cohen's d) was used to examine the clinical effect of the intervention [23].

\section{Results}

The experimental group consisted of five male and three female subjects with the mean age of seven years and 10 months and the control group included four male and four female subjects with the mean age of seven years and nine months. There was no significant difference in age between the two groups. Comparison of the

Table 1. Comparison of post-test scores of the two groups

\begin{tabular}{|c|c|c|c|c|}
\hline \multirow{2}{*}{ Test } & \multicolumn{2}{|c|}{ Post-Test (Mean \pm SD) } & \multirow{2}{*}{$\mathbf{P}$} & \multirow{2}{*}{ Effect Size } \\
\hline & Case & Control & & \\
\hline Eye-motor coordination & $18.88 \pm 4.79$ & $16.75 \pm 3.99$ & 0.36 & 0.48 \\
\hline Figure-ground & $13.88 \pm 4.12$ & $11.25 \pm 7.14$ & 0.42 & 0.45 \\
\hline Form constancy & $7.5 \pm 3.2$ & $7.75 \pm 4.52$ & 0.95 & 0.06 \\
\hline Position in space & $4.13 \pm 2.03$ & $3.88 \pm 1.72$ & 0.78 & 0.13 \\
\hline Spatial relationships & $5.75 \pm 0.88$ & $4 \pm 2.44$ & 0.13 & 0.95 \\
\hline Error in providing response & $9.38 \pm 5.15$ & $12.75 \pm 11.6$ & 0.87 & 0.37 \\
\hline Deleted response & $5.88 \pm 3.18$ & $8.63 \pm 6.25$ & 0.31 & 0.55 \\
\hline Correct response & $134.75 \pm 5.52$ & $128.63 \pm 16.39$ & 0.46 & 0.50 \\
\hline
\end{tabular}


Table 2. Comparison of pre- and post-test scores in the control group

\begin{tabular}{|c|c|c|c|}
\hline \multirow{2}{*}{ Test } & \multicolumn{2}{|c|}{ Control (Mean \pm SD) } & \multirow{2}{*}{$\mathbf{P}$} \\
\hline & Pre-Test & Post-Test & \\
\hline Eye-motor coordination & $13.75 \pm 5$ & $16.75 \pm 3.99$ & 0.16 \\
\hline Figure-ground & $9.5 \pm 6.78$ & $11.25 \pm 7.14$ & 0.19 \\
\hline Form constancy & $6.25 \pm 5.49$ & $7.75 \pm 4.52$ & 0.16 \\
\hline Position in space & $4 \pm 2.07$ & $3.88 \pm 1.72$ & 0.91 \\
\hline Spatial relationships & $3.63 \pm 2.44$ & $4 \pm 2.44$ & 0.18 \\
\hline Error in providing response & $16.13 \pm 16.09$ & $12.75 \pm 11.64$ & 0.48 \\
\hline Deleted response & $10.88 \pm 9.89$ & $8.63 \pm 6.25$ & 0.44 \\
\hline Correct response & $123 \pm 22.86$ & $128.63 \pm 16.39$ & 0.26 \\
\hline
\end{tabular}

Iranian Rehabilitation Journal

mean scores of the experimental and control groups in the pre-test with the Mann-Whitney nonparametric test indicated that no difference between the groups in the visual perception and attention score before the intervention.

The tests were repeated after the completion of intervention; however, the 0.95 Cohen's $d$ in the 5th subtest revealed a significant effect of the intervention on the ability to understand spatial relationships in the experimental group compared with the control group after the intervention. Based on the Cohen's d effect size measure, which is capable of detecting the clinical impact of interventions in very little or very large sample sizes, the effects of $0.2>, 0.2-0.49$, $0.5-0.79$, and $>0.8$ showed an insignificant, low, medium, and high effects. Accordingly, the more average impact of intervention on attention and concentration was observed in the experimental group, compared with the control group by the number of fewer eliminated responses and the more correct answers (Table 1).

Table 3. Pre- and post-test scores in the experimental group

\begin{tabular}{|c|c|c|c|c|}
\hline \multirow{3}{*}{ Test } & \multicolumn{2}{|c|}{ Examination } & \multirow{3}{*}{$\mathbf{P}$} & \multirow{3}{*}{ Effect Size } \\
\hline & \multicolumn{2}{|c|}{ Mean $\pm S D$} & & \\
\hline & Pre-Test & Post-Test & & \\
\hline Eye-motor coordination & $17.5 \pm 2.26$ & $18.88 \pm 4.79$ & 0.52 & 0.36 \\
\hline Figure-ground & $12 \pm 3.92$ & $13.88 \pm 4.12$ & 0.03 & 0.46 \\
\hline Form constancy & $4.5 \pm 2.39$ & $7.5 \pm 3.2$ & 0.10 & 1.06 \\
\hline Position in space & $5 \pm 1.06$ & $4.13 \pm 2.03$ & 0.19 & 0.53 \\
\hline Spatial relationships & $5.5 \pm 1.77$ & $5.75 \pm 0.88$ & 0.91 & 0.17 \\
\hline Error in providing response & $6 \pm 5.18$ & $9.38 \pm 5.15$ & 0.04 & 0.65 \\
\hline Deleted response & $7.88 \pm 6.83$ & $5.88 \pm 3.18$ & 0.67 & 0.37 \\
\hline Correct response & $136.13 \pm 11.65$ & $134.75 \pm 5.52$ & 0.72 & 0.15 \\
\hline
\end{tabular}


Comparison of pretest and post-test scores in the control group using the nonparametric Wilcoxon test showed no significant difference between the pretest and post-test scores (Table 2).

However, in the intervention group, results of the Wilcoxon test showed a significant difference between the pretest and post-test scores of the intervention group in the subtest of figure-ground perception. Cohen's d effect size measure also showed that the intervention had a modest impact on the perception of position in space and the significant impact on the form constancy perception of the experimental group (Table 3 ).

\section{Discussion}

The current study was the first investigation on the effectiveness of a computer game on the improvement of visual-motor coordination in children with hearing impairments. The short-term impact of SHOFER game was investigated in the current study and it was found that this game may affect some elements of motor coordination. Despite the lack of a significant difference among the results, clinical evidence (Cohen's d effect size) suggested that the SHOFER game may cause visual-motor coordination improved through enhancing perception of position in space and the form constancy in the experimental group.

The game also had a significant impact on the ability to perceive the spatial relationships in the experimental group compared with the control group. These results are in line with those of previous studies that showed the effectiveness of computer games [13-15]. The type of cognitive and perceptual skills required for playing action, driving, maze, and puzzle genres can influence cognitive skills and spatial perception [24]. Spatial perception, as part of visual perception, is the ability to navigate in the environment and identify the right, left, front, back, and top directions, and understand the status of objects surrounding $[20,25]$.

The lack of significant results can be attributed to the genre of the computer game used for the current study. Driving is a popular and playable genre due to easy gameplay, recognizable goals, and a fewer number of elements that should be controlled. However, these features make this genre easier compared to more complex genres such as action that have less opportunity for technical play performance that might reduce the impact of SHOFER game [26].
The impact of racing genre on visual skills could be due to the game speed, the number of objects to follow, and the emphasis on environmental processing. In general, it can be concluded that the type and genre of computer games, targets, and characters defined in the game make considerable differences in their effect [27]. Selecting the considered objects, the pursuit of multiple objects, and the complex three-dimensional environment are the effective characters in computer games affecting attention, which in turn, can affect the visualmotor skill. Additionally, the need for coordination to achieve goals and navigate can be of other influential characters of these games on visual-motor performance and spatial recognition [24].

SHOFER game with Iranian roads and cars, in the first place, was well-received by the experimental group and the reality of the game environment can be cited as its attractiveness. However, lack of exciting characters and the monotonous rhythm of driving a car on the road diminished its initial attractiveness. On the other hand, one of the features of the SHOFER game was the indigenous music as the background music that the experimental group was deprived of hearing it. Xiaoqing declared that the existence of the background music increases the sense of immersion and the realization of the game experience that ultimately leads to the attractiveness and maintenance of the player's constant attention to the game [28]

The experimental group expressed the concepts of "win" and "victory in the race" and the need for progress in order to get a new car by shaking head and hands and using the sign language. Despite encountering the game for the first time, few children in the experimental group had played the game only in trial and error mode. These children were negligent and bored in the study, and their teacher said that they usually had poor concentration in the classroom. However, most subjects in the experimental group showed planning behaviors during the game. They got upset and angry about their failure, and each time they spoke with the sign language "I understand" or "I win this time". It is likely that planning ability could be promoted as a training-based performance in the game for the studied students [29].

The strength of the current study was the utilization of a commercial and indigenous game for the health purposes amongst children with hearing impairment. However, the current study could serve as a pilot study. The major limitation was the small sample size recruited with no sample size calculation method. Although the results indicated the positive effects of SHOFER computer game 
on some aspects of visual-motor coordination, further research in needed to prove these findings. The impact of SHOFER game was also evaluated immediately after the end of the intervention and was not followed. It is therefore recommended that similar studies with followup strategies and larger sample sizes be performed.

\section{Conclusion}

Overall, results of the current study showed that SHOFER computer game could be effective on enhancing different aspects of the visual-motor coordination. Thus, therapists can advise this commercial game for their clients with hearing problems. Additionally, the acceptability of a computer game among children with hearing impairment can help them to develop their potential for learning.

\section{Ethical Considerations}

\section{Compliance with ethical guidelines}

The current article was extracted from the master's thesis of Zahra Norouzi Tabrizi Nejad majored in the field of health information technology of Shiraz University of Medical Sciences (Code: 1396-01-07-15379). All participants were allowed to quit the study at any stage. The school director and teachers and the children's parents were informed that participation in the study was voluntary and the study would not cause any harm to the children. The study protocol was registered with the Iranian Registry of Clinical Trials (IRCT20140618018150N3). Parent informed consent in the current study was approved by the local Ethics Committee of Shiraz University of Medical Sciences (IR.SUMS. REC.1396.178). In addition, the confidentiality and privacy of information was preserved throughout the steps of the study. A moral assurance was also given to the Department of Exceptional Education in Ahvaz to provide them with a copy of the results.

\section{Funding}

The research was funded by the Vice-Chancellor of Science and Technology Affairs of Shiraz University of Medical Sciences, Iran.

\section{Authors' contributions}

Conceptualization: Zahra Norouzi Tabrizi Nejad; Methodology: All authors; Investigation, writing original draft: Zahra Norouzi Tabrizi Nejad, Seyed Alireza Derakhshanrad; Writing-review and editing: All authors; Funding acquisition: Rita Rezaee; Supervision; Rita
Rezaee, Seyed Alireza Derakhshanrad, Ali Mohammad Hadianfard; Project administration: All authors.

\section{Conflict of interest}

The authors declared no conflict of interest.

\section{Acknowledgments}

The authors are grateful to the honest cooperation of the director and teachers of the exceptional school and all the children that participated in the study.

\section{References}

[1] World Health Organization (WHO). Deafness and hearing loss [Internet]. 2018 [updated 15 March 2018]. Available from: http://www.who.int/mediacentre/factsheets/fs300/en/

[2] McPhillips M. Motor difficulties and mental health in children who are deaf. Developmental Medicine \& Child Neurology. 2015; 57(10):893-4. [DOI:10.1111/dmcn.12831] [PMID]

[3] Fellinger MJ, Holzinger D, Aigner M, Beitel C, Fellinger J. Motor performance and correlates of mental health in children who are deaf or hard of hearing. Developmental Medicine \& Child Neurology. 2015; 57(10):942-7. [DOI:10.1111/ dmcn.12814] [PMID]

[4] Wiegersma P, Velde AV. Motor development of deaf children. Journal of child Psychology and Psychiatry. 1983; 24(1):103-11. [DOI:10.1111/j.1469-7610.1983.tb00107.x] [PMID]

[5] Hanson GH, Hancock BB, Kopra LL. Relationships among audiological status, linguistic skills, visual-motor perception, and academic achievement of deaf children. Austin: University of Texas at Austin; 1969 .

[6] Shojaie R. [The analysis of visual/motional skill in hearingimpaired children (Persian)]. Journal of Exceptional Education. 2012; 3(111):67-72

[7] Buckner MK. Eye hand coordination [Internet]. 2018 [Updated 22 September 2018]. Available from: http://therapystreetforkids.com/EyeHandCoord.html

[8] Roux Tl. Visual motor integration [Internet]. 2018 [Updated 29 August 2018]. Available from: https://www.ot-momlearning-activities.com/visual-motor-integration.html

[9] Kid Sense. Visual Perception [Internet]. 2018 [Updated 22 November 2018]. Available from: https://childdevelopment.com. $\mathrm{au} /$ areas-of-concern/fine-motor-skills/visual-perception/

[10] Green CS, Bavelier D. Action-video-game experience alters the spatial resolution of vision. Psychological Science. 2007; 18(1):8894. [DOI:10.1111/j.1467-9280.2007.01853.x] [PMID] [PMCID]

[11] Li L, Chen R, Chen J. Playing action video games improves visuomotor control. Psychological Science. 2016; 27(8):1092108. [DOI:10.1177/0956797616650300] [PMID] 
[12] Anguera JA, Boccanfuso J, Rintoul JL, Al-Hashimi O, Faraji $\mathrm{F}$, Janowich J, et al. Video game training enhances cognitive control in older adults. Nature. 2013; 501(7465):97-101. [DOI:10.1038/nature12486] [PMID] [PMCID]

[13] Kovess-Masfety V, Keyes K, Hamilton A, Hanson G, Bitfoi A, Golitz $\mathrm{D}$, et al. Is time spent playing video games associated with mental health, cognitive and social skills in young children? Social Psychiatry and Psychiatric Epidemiology. 2016; 51(3):349-57. [DOI:10.1007/s00127-016-1179-6] [PMID] [PMCID]

[14] Griffiths M. Video games and health. British Medical Journal. 2005; 331(7509):122-3. [DOI:10.1136/bmj.331.7509.122] [PMID] [PMCID]

[15] Sandlund M, McDonough S, Häger-Ross C. Interactive computer play in rehabilitation of children with sensorimotor disorders: a systematic review. Developmental Medicine \& Child Neurology. 2009; 51(3):173-9. [DOI:10.1111/j.14698749.2008.03184.x] [PMID]

[16] Mader S, Natkin S, Levieux G. How to analyse therapeutic games: The player/game/therapy model. International Conference on Entertainment Computing. Berlin: Springer; 2012. [DOI:10.1007/978-3-642-33542-6_17]

[17] Cano S, Collazos CA, Aristizábal LF, Gonzalez CS, Moreira F. Towards a methodology for user experience assessment of serious games with children with cochlear implants. Telematics and Informatics. 2018; 35(4):993-1004. [DOI:10.1016/j. tele.2017.09.011]

[18] SHOFER race driver: STEAM [Internrt]. 2015 [Updated 22 September 2015]. Available from: http://store.steampowered.com/app/367700/SHOFER_Race_Driver/

[19] Iran Computer \& Video Games Foundation. SHOFER. Tehran: Iran Computer \& Video Games Foundation; 2015.

[20] Frostig M, Lefever W, Whittlesey J. Frostig developmental test of visual perception, diagnosis and treatment [Persian] Tehran: Farvardin; 1996.

[21] Noughabi R, Dortaj F. [Standardization, validity, and reliability of frostig developmental test of visual perception among first and second grade elementary school students in Tehran (Persian)]. Journal of Applied Psychology. 2007; 1(3):253-68.

[22] Hadyanfar H, Najjarian B, Shokrkon H, Mehrabi M. [Construction of Persian version of continuous performance test (Persian)]. Journal of Psychology. 2000; 4(4):388-404.

[23] Cohen J. Statistical power analysis for the behavioral sciences. Cambridge, Massachusetts: Academic Press; 1977.

[24] Spence I, Feng J. Video games and spatial cognition. Review of General Psychology. 2010; 14(2):92-104. [DOI:10.1037/a0019491]

[25] Asliazad M, Abedi A, Iarmohammadian A. [The effectiveness of spatial relations perception training on the mathematics performance of boy students with math learning disability (Persian)]. Psychology of Exceptional Individuals. 2015; 5(17):113-31.

[26] Araujo TB, Silveira FR, Souza DL, Strey YT, Flores CD, Webster RS. Impact of video game genre on surgical skills development: A feasibility study. The Journal of Surgical Research. 2016; 201(1):235-43. [DOI:10.1016/j.jss.2015.07.035] [PMID]

[27] Rosas R, Nussbaum M, Cumsille P, Marianov V, Correa M, Flores $\mathrm{P}$, et al. Beyond nintendo: Design and assessment of educational video games for first and second grade students. Computers \& Education. 2003; 40(1):71-94. [DOI:10.1016/ S0360-1315(02)00099-4]

[28] Per X, Zhang J. The influence of strategy video game and its background music on cognitive control. EC Psychol-ogy and Psychiatry. 2017; 2(1):15-25. [DOI:10.15761/MHAR.1000125]

[29] Perzov A, Kozminsky E. The effect of computer games practice on the development of visual perception skills in kindergarten children. Computers in the Schools. 1989; 6(3-4):113-22. 